\title{
Menstrual Blood
}

National Cancer Institute

\section{Source}

National Cancer Institute. Menstrual Blood. NCI Thesaurus. Code C78740.

The discharged blood from the uterus during the reproductive years. The discharge occurs approximately once a month. 